\title{
Natural Language Processing and its Use in Education
}

\author{
Dr. Khaled M. Alhawiti \\ Computer Science Department, Faculty of Computers and Information technology \\ Tabuk University, Tabuk, Saudi Arabia
}

\begin{abstract}
Natural Language Processing (NLP) is an effective approach for bringing improvement in educational setting. Implementing NLP involves initiating the process of learning through the natural acquisition in the educational systems. It is based on effective approaches for providing a solution for various problems and issues in education. Natural Language Processing provides solution in a variety of different fields associated with the social and cultural context of language learning. It is an effective approach for teachers, students, authors and educators for providing assistance for writing, analysis, and assessment procedures. Natural Language Processing is widely integrated with the large number of educational contexts such as research, science, linguistics, e-learning, evaluations system, and contributes resulting positive outcomes in other educational settings such as schools, higher education system, and universities. The paper aims to address the process of natural language learning and its implication in the educational settings. The study also highlights how NLP can be utilized with scientific computer programs to enhance the process of education. The study follows qualitative approach. Data is collected from the secondary resources in order to identify problems faced by the teachers and students for understanding the context due to obstacles of language. Results provide effectiveness of linguistic tools such as grammar, syntax, and textual patterns that are fairly productive in educational context for learning and assessment.
\end{abstract}

Keywords-Natural Language Processing; education; application; e-learning; scientific studies; educational system

\section{INTRODUCTION}

Natural language process is an effective process to assist students in the process of scientific learning. Implementing NLP in the educational setting not only helps in developing effective language process, but it is also significant for enhancing the academic performance. The NLP techniques follow the approach of the natural process of language acquisition integrated with the scientific approach of using computer programs.

\section{A. Outline of the Study}

This study is based on "Application of Natural Language Processing in Education". The first section of this study will provide an "introduction" to the topic that discuss and define the background of natural language learning process. This part will also focus on the aims and objectives of the study. The second sections contain materials discussing the Natural language processing Arabic language and its implementation in the educational framework. Next section is comprised of procedure and method of the study, Material and methods provide sufficient details to allow the work to be reproduced. Results provide detailed analysis of the data collected from the study. Discussion part of the study is comprised of detailed analysis and valuation of the results based on the data obtained from the research study. The last section of the study is conclusion, which is comprised of summary of the study and useful implementations and recommendations for further research.

\section{B. BackGround of the Study}

Natural language processing NLP is a major factor associated with the branch of science, which focus on the development and improvement in the process of learning. NLP provides theoretical grounds to assist in the process of developing techniques and effective approaches for providing assistance in the scientific learning by utilizing the effective theories and approaches. NLP can be effectively applied in the education for promoting the language learning and enhancing the academic performance of the students. [1] Natural language processing assists in developing effective process of learning in the educational setting by developing scientific approaches, which can assist in the process of using computer and internet for improvement the learning. In order to provide assistance, there are a number of different computer programs and effective language learning approaches to ensure that students can easily develop understanding of education in the natural settings. This is based on utilizing the effective and efficient language learning process in the natural settings [1]. NLP utilize the natural language process and utilize this process for developing effective approaches in order to bring improvement in the educational settings.

The approach in NLP is more focused on developing educational software systems and educational strategies that can assist in utilizing the natural languages for education, for example, e-rater and Text Adaptor [2]. The software systems with the NLP have the ability to identify the process of language learning in natural settings.

The Natural language processing is also an effective approach for developing an efficient system of managing linguistic input in the natural settings through various words, sentences, and texts. The Natural Language processing also use various grammatical rules and linguistic approaches such as derivations, infections, grammar tenses, semantic system, lexicon, corpus, morphemes, tenses etc. All these effective approaches can be applied in the educational settings in order to ensure that students can develop better understanding of the educational material and curriculum. 
Natural Language Processing is a widely recognized area in the language learning all over the world. It is successfully implemented in different languages as an effective way for bringing improvement in the educational systems. English is the most commonly recognized language in most of the researched studies, which reveals it's effective of utilizing natural language learning process in educational process. NLP is also effective approach for bringing improvement in the educational system in Arab countries [3]. Despite that there are various approaches for bringing improvement in the social and educational settings; NLP is the best suitable approach, in which natural language processing use to create NLP tools to promote the education. These tools are based on utilizing various effective approaches for assisting in the process of education at college and university level. This requires developing the tools and corpus resources for the educational system in Arabic language.

\section{Aims and Objectives}

The major aims and objectives of this study is to

- Understand the natural language learning processing.

- The objective of this study is to apply the natural language processing NLP in educational setting.

\section{MATERIAL AND METHODS}

This study is based on qualitative approach. Method of data collection is based on gathering information from the secondary resources and analysis of the theories, which support and assist in understanding the natural language process and its implementation in the education. Various problems faced by the teachers and students for understanding the context due to obstacles of language. The use of effective linguistic tools such as grammar, syntax, and textual patterns are very effective for learning and assessment of text. The study is based on a qualitative approach. Method of data collection is based on gathering information from the secondary resources and analysis of the theories, which support and assist in understanding the natural language process and its implementation in the education. Various problems faced by the teachers and students for understanding the context due to obstacles of language. The use of effective linguistic tools such as grammar, syntax, and textual patterns are very effective for learning and assessment of text. [4]

\section{A. NLP and Educational Setting}

There are a number of different effective approaches in the NLP, which assist in educational settings such as role of empirical data, corpora, and other such linguistic aspects, which are essential and effective for the process of language learning. Corpora are very effective, which provides a large number of computational data for spoken and written language. For example, in British English, BNC (the British National Corpus) provides a large data about the vocabulary usage [4,5]. The large collection of information provides sufficient data regarding the usage of words, which assist enhancing the information and academic skills of the students.

There are various effective approaches, which are effective for managing patterns of grammar and other linguistic approaches. NLP is also an effective technique for assessment process to enhance the ability of students to identify the relationships of different words and the use of such words in the search engine for generating treasure [5].Therefore; it is an effective approach, which allows learners and teachers to use these words more efficiently. The assessment procedure requires entering correct information in the text in order to enter in the next level. NLP assessment allows analysis of the students' information by matching it with the requirement of the content [6].

\section{B. Tools and Methods}

Having access to a variety of tools, NLP enjoys the implementation in multiple fields such as laboratory, faculty of information, e-learning, and in the field of education. Availability of search engines also provide sufficient information for information search, but language constraints are a major issue for majority of students, which hinders the process of language learning through electronic sources and online material available on internet. The natural language learning processing is also associated with the understanding of various effective linguistic tools such as grammatical construction, syntax, sentence composition, etc [7].

The application of NLP in the area of e-learning is an effective approach, especially its application in the area of educations. The natural language processing can assist students in developing general understanding of the cognitive and psychological perspectives that play an important role in the language acquisition. In the educational process, natural language processing can be implemented effectively to ensure that there are various positive attributes of this approach such as specification of the synchronous or asynchronous mode. [8]

The methods for the implementation of NLP in education requires using the approach of e-learning or the utilization of teaching material for bringing improvement for further development $[8,9]$. The method or the process of this study also requires implementation of the effective approaches and utilization of the language resources for bringing improvement in the system of education. There are also a large number of tools and methods, which assist in utilization of language technology such as linguistics software systems, which are very effective for managing and dealing with the need to bring improvement in the educational system [9].

The implementation of language tools can assist in developing better understanding of the content such as better understanding of material while reading, development of the reading text and material. There are two sides of the educational system: learning and teaching. Thus, tools for teaching and learning are both effective for assisting the process of educations such as websites, publications, digital libraries, e-books, podcasts, and scientific materials [10]. This is one of the most effective approaches, which allows students and teachers to focus and to explore more in the field of study. There are various effective methods to implement NLP in the educational settings such as classification and categorization of different sources in the learning perspectives. This can assist in identification of the authentic sources and avoiding the use of unreliable resources. 
Another method to assist language learners is through the process of enabling students to concentrate on their course material and content of the given topic. This approach is based on matching the course content knowledge of learners; this method is derived from the assessment procedure of NLP. For example, students can be asked to write an essay, which matches their curse content with the current information gathered from the online source. [11]

\section{Theory}

Reference [2] discussed emerging opportunities for bringing improvement in the natural language process NLP and its effectiveness for developing various educational tools such as for reading and writing content. According to Reference [2] NLP provides theoretical grounds and practical implementations for the technology based computer systems. Advancement of computer technology and the increase in approach of second language learning has resulted in the trend of using language as a major tool for providing assistance in the educational settings. According to Burstein, "the general lack of computer based technology proved to be an obstacle early on" [2].NLP allows the assessment of readability and text quality by analyzing the text quality especially $n$ terms of the text analysis.

The linguistics aspects in the educational context can assist in managing and dealing with the complexities of reading and writing. This can be done with the approach of analyzing syntactic and morphological factors. Motivation in language learning is an effective approach, which can also be applied to enhance the educational practices and academic performance of students [12]. Teachers can motivate students to focus and develop understanding of their content; it also requires utilizing effective strategies for the students to set the programs in various domains. The linguistic tools assist in the process of evaluating and managing the problems of lexical and syntactic composition in writing and develop the understanding of content $[13,14]$. Learning and developing better understanding of language can also assist in better understanding of the content. The theory of natural acquisition of language is also an effective approach to explain the ground for developing content knowledge acquisition.

\section{RESULTS AND DISCUSSIONS}

\section{A. Innovatiove Education Applications of NLP}

Text Evaluator also known as Source Rater is a trending tool that represent a trending concept for modeling text complexity, created to help assist developers assess source material for use in creation of new reading comprehension passages and items. This tool unites a large, cognitively based features set with sophisticated psychometric approaches in order to present text complexity categorizations that are highly associated with categorization provided by experienced educators.

Language Muse is another example educational application of NLP, using a web-based application for instructional authoring and projected to support K12 instructors in the creation of English-language learners' curriculum material. The linguistic feedback given by this application highlights sentence structures, vocabulary, and discourse relations that may be identified in classroom texts that may be unfamiliar to ELLs. Automated Generation of test items is another application towards the use of Natural Language Processing. [14]

\section{B. Natural Language Processing and Edcation}

Natural Language processing has various applications for educational purpose. It is very significant to develop new software systems and advanced techniques in the educational settings. The major purpose of using NLP in educational setting is to bring improvement in the educational system by implementing efficient and effective policies, which can assist in utilizing advance technologies for the bringing improvement in the educational system. For example, application of NLP in education for e-learning is very significant approach, which assist in producing educational material with technological development. Another significance of NLP application is the participation of both teachers and students. There are a number of various electronic, online sources available in English language, which assists students and teachers to access materials. Apart from the convenience of availability of large number of online resources, another major concern is associated with the increase in use of blogs, Wikipedia, and unreliable resources. This requires intelligent automatic processing for preventing the use of such unreliable resources and promoting the use of authentic resources. Application of NLP in education is also effective for mining, information retrieval, and quality assessment.

\section{Relationship between Language and Text}

Results based on the secondary sources and theoretical grounds reveal that text and technology have close relationships. Students cannot understand content without proper understanding of the language. Without understanding of language, it is not possible for the learners to understand and retain information. The process of natural language acquisition is one of the most important strategies that can assist in the process of language acquisition. The natural language acquisition process along with the motivation from teachers can serve as a major source for bringing improvement in the academic process of the students. Teachers and learners can both focus on the strategies, which are efficient for utilizing language in educational setting. For example, research studies reveal that context analysis is not possible without developing the understanding of text.

According to Reference [15] the implementation of natural language processing for education reveals that teachers and educators can follow NLP approach for designing and planning curriculum. This can also assists students to follow same strategic approach for learning and understanding the content. The authors of texts and content can use this approach for storing and coding the information for the students, and students follow same approach and linguistic composition for decoding the text. Therefore, knowledge of natural language process is effective for educators, teachers, authors, and students for better learning and understanding of the content, and improvement of writing skills. The results also suggest that this approach is effective for its application in the assisting students to study material from web resources, especially in the e-learning. 


\section{Application of Natural Language Processing in Education}

There are a number of different effective approaches, which assist in the process of e-learning and using web based current information related to the educational course and curriculum. E-learning applications and tools provide assistance for the learners to improve their education. Teachers also assist their students for enhancing their skills and knowledge for getting current information using the online resources, which assists in getting information from the online sources. NLP is also very effective for providing knowledge and information to the students for application of e-learning and NLP in understanding and dealing with the need of analyzing text. Understanding of text is based on the development of research-based analysis of the general and contextual learning.

Based on the research outcome, it is clear that students' output can be increased by implementing the NLP in the education. NLP is very effective approach for developing the understanding of students in the natural settings and assessing the information available from the various sources. The better understanding of information and ability to access the information from large amount of data available on websites and other online sources can assist in generating and gathering information. Therefore, based on the results and effectiveness of NLP in the educational context, it is clear that NLP can be effectively applied for academic writing, assessment purposes, writing tests questions, and utilizing automatic writing systems for preparation of objective tests etc. [15]

The application of NLP in education system is also very effective for analysis of errors in objective assessments and for the assessment of essays. Various linguistic approaches and tools can be utilized for analyzing the errors such as grammatical and stylistic errors. Teachers can easily mark these errors in the papers of students. There are various effective grammar checkers and evaluation sources, which assist in resolving the problems of dealing with the current process of learning. Teachers can use NLP for assessment of multiple-choice questions and analysis of grammatical pattern in the text that needed to be analyzed. The application of Standard e-learning method is very effective in order to ensure that student can efficiently apply the data in the e-learning system. This approach is not only affective for its application in assessment, but it is also effective for writing purposes such as writing material for digital libraries, websites, and various other sources.

\section{CONCLUSIONS}

In conclusion, Natural Language Processing and its Educational Application provide a perfect solution to the various problems and barriers in the educational system, which result in affecting the academic progress and learning of the students. Language is one of the major concerns for the students. NLP with an effective approach for assisting the progress and improvement in the learning ability of students based on development and implementation of various effective tools, assist writing, learning, and assessment of texts, such as use of search engines, electronic resources and analysis of grammatical construction, syntax, sentence composition, etc. All these are the effective techniques, which can be utilized to develop the structural framework for analysis of texts.
The use of grammar, syntax, and sentence composition can be efficiently utilized through linguistics software systems such as grammar checkers, which are saves times and provides assistance for both teachers and learners. Therefore, there is need for developing effective approach for the social and cultural perspectives. Implementation of NLP is also effective for using the e-learning approach in order to understand and learn from the data available from the electronic sources. There are also future implementations of this research, which can assists in identifying the complex pattern in language. Further research can be conducted to identify its impact in individual learning, understanding of context, and effectiveness of NLP in writing and assessment procedure.

\section{REFERENCES}

[1] Nadkarni, P. M., Ohno-Machado, L., \& Chapman, W. W. (2011). Natural language processing: an introduction. Journal of the American Medical Informatics Association, 18(5), 544-551

[2] Burstein, J. (2009). Opportunities for natural language processing research in education. In Computational Linguistics and Intelligent Text Processing (pp. 6-27). Springer Berlin Heidelberg. http://link.springer.com/chapter/10.1007/978-3-642-00382-0_2\#page-1

[3] Habash, N. Y. (2010). Introduction to Arabic natural language processing.Synthesis Lectures on Human Language Technologies, 3(1), $1-187$.

http://www.morganclaypool.com/doi/abs/10.2200/s00277ed1v01y20100 8 hlt010

[4] Liu, K., Hogan, W. R., \& Crowley, R. S. (2011). Natural language processing methods and systems for biomedical ontology learning. Journal of biomedical informatics, 44(1), 163-179.

[5] De Vries, M. H., Monaghan, P., Knecht, S., \& Zwitserlood, P. (2008). Syntactic structure and artificial grammar learning: The learnability of embedded hierarchical structures. Cognition, 107(2), 763-774. http://dl.acm.org/citation.cfm?id=1967546

[6] Pascual-Nieto, I., Santos, O. C., Perez-Marin, D., \& Boticario, J. G. (2011, July). Extending Computer Assisted Assessment Systems with Natural Language Processing, User Modeling, and Recommendations Based on Human Computer Interaction and Data Mining. In IJCAI Proceedings-International Joint Conference on Artificial Intelligence (Vol. 22, No. 3, p. 2519)

[7] Wahl, H., Winiwarter, W., \& Quirchmayr, G. (2010, November). Natural language processing technologies for developing a language learning environment. In Proceedings of the 12th International Conference on Information Integration and Web-based Applications \& Services (pp. 381-388). ACM.

[8] de-la-Fuente-Valentín, L., Carrasco, A., Konya, K., \& Burgos, D. (2013). Emerging Technologies Landscape on Education. A review. International Jorunal of Interactive Multimedia and Artificial $\begin{array}{lrr}\text { Intelligence, } & 2(3), & 55-70 .\end{array}$ http://dialnet.unirioja.es/descarga/articulo/4426252.pdf

[9] Felix, U. (2005). E-learning pedagogy in the third millennium: the need for combining social and cognitive constructivist approaches. ReCALL, 17(01), 85-100. http://journals.cambridge.org/action/displayAbstract?fromPage=online\& aid $=305554 \&$ fileId $=$ S0958344005000716

[10] Benson, P. (2013). Teaching and researching: Autonomy in language learning. Routledge. http://books.google.com/books?id=ZoarAgAAQBAJ\&printsec=frontcov er

[11] Castellani, S., Kaplan, A., Roulland, F., \& Roux, C. (2010). U.S. Patent No. 7,797,303. Washington, DC: U.S. Patent and Trademark Office. http://www.google.com/patents/US7797303

[12] Shi, C., Verhagen, M., \& Pustejovsky, J. (2014). A Conceptual Framework of Online Natural Language Processing Pipeline $\begin{array}{llll}\text { Application. } & \text { COLING } & \text { 2014, }\end{array}$ http://www.aclweb.org/anthology/W/W14/W14-52.pdf\#page=63 
[13] Najeeb, M. M., Abdelkader, A. A., \& Al-Zghoul, M. B. (2014). Arabic Natural Language Processing Laboratory serving Islamic Sciences. International

http://thesai.org/Downloads/Volume5No3/Paper_16-

Arabic_Natural_Language_Processing_Laboratory_serving_Islamic_Sci ences.pdf
[14] Richards JC, Rodgers T (2001) Approaches and Methods in Language Teaching. Second Edition. New York: Cambridge University Press

[15] Van den Branden (2012) Task-based language education. In: Burns A, Richards JC, The Cambridge Guide to Pedagogy and Practice in Language Teaching. New York: Cambridge University Press, 140-48. 